\title{
Ion-Acoustic Higher Order Non-Linear Structures in Quantum Dusty Plasma
}

\author{
Md Manir Hossain ${ }^{1}$, Monirul Hasan ${ }^{1}$, Md Asaduzzaman², Md Mahfuzul Haque ${ }^{1}$ \\ ${ }^{1}$ Department of Electrical and Electronic Engineering, Bangladesh University of Business and Technology, \\ Dhaka, Bangladesh \\ ${ }^{2}$ Institute of Natural Science, United International University, Dhaka, Bangladesh \\ Email: monirul.h@bubt.edu.bd
}

Received June 3, 2013; revised July 5, 2013; accepted August 1, 2013

Copyright (C) 2013 Md Manir Hossain et al. This is an open access article distributed under the Creative Commons Attribution License, which permits unrestricted use, distribution, and reproduction in any medium, provided the original work is properly cited.

\begin{abstract}
The prominent features of higher order nonlinear ion-acoustic waves involving quantum corrections in an unmagnetized quantum dusty plasma are revisited with the theoretical framework of Hossain et al. [1]. The fluid model is demonstrated here by its constituent inertial ions, Fermi electrons with quantum effect, and immovable dust grain with negative charge. We have used the ideology of Gardner equation. The well-known RPM method is employed to derive the equation. Indeed, the basic features of quantum dust ion-acoustic Gardner solitons (GSs) are pronounced here. GSs are shown to exist for the value of dust to ion ratio around 2/3 which is valid for space plasma [2], and are different from those of K-dV (Korteweg-de Vries) solitons, which do not exist for the value around 2/3. The implications of our results are suitable for cosmological and astrophysical environments.
\end{abstract}

Keywords: Negative Dust; Quantum Plasma; Fermi Electron; Modified K-dV Equation; Gardner Solitons; RPM

\section{Introduction}

Quantum plasmas have attracted a great deal of attention because of their potential applications in dense plasma particularly in different astrophysical and cosmological systems [3-5] (e.g. interstellar or molecular clouds, planetary rings, comets, interior of white dwarf stars, etc.), in nanostructures [6], in microelectronic device [7] as well as in the next-generation intense laser [8]. Many authors have proposed some theories including the quantum corrections to the quantum plasma echoes [9], the self-consistent dynamics of Fermi gases [10], quantum beam instabilities [11], wave interactions in quantum magnetoplasmas [12], classical and quantum kinetics of the Zakharov system [13], quantum corrections to the Zakharov equations [14], expansion of quantum electron gas into vacuum [15], quantum ion acoustic waves [16], quantum Landau damping [17], magnetohydrodynamics of quantum plasmas [18], etc. Quantum plasmas have extremely high plasma number densities and low temperatures. At extremely low temperatures, the thermal de Broglie wavelength becomes comparable to the interelectron distance and the electron temperature becomes comparable to the electron Fermi temperature $\left(T_{\mathrm{Fe}}\right)$ and the electrons follow Fermi
Dirac distribution law. In this condition, quantum mechanical effects are expected to play a significant role in the behavior of charged particles [19-21]. As electrons are lighter than ions, the quantum behavior of electron is reached faster than ions. The dust particles are quite common in various plasma systems. The inclusion of immobile charged dust in electron-ion plasmas leads to introduce a new mode. Shukla and Silin [22] have first theoretically shown the existence of low-frequency dust ion-acoustic (DIA) waves in a dusty plasma, which was latter observed in laboratory experiments [23,24]. The phase speed of the DIA waves is much smaller (larger) than electron (ion) thermal speed. The inertia is provided by the ion mass while the restoring force comes from the electron thermal pressure. These waves differ from usual ion-acoustic waves [25] due to the conservation of equilibrium charge density $n_{e 0}+Z_{d} n_{d 0}-n_{i 0}=0$ and the strong inequality $n_{e 0} \ll n_{i 0}$, where $n_{s 0}$ is the particle number density of the species $s$ with $s=e$ for electrons, $i$ for ions and $d$ for dust, $Z_{d}$ is the number of electrons residing onto the dust grain surface, and $e$ is the magnitude of an electronic charge. Therefore, a dusty plasma can not support the usual ion-acoustic waves, but 
can do the DIA waves of Shukla and Silin [22]. The nonlinear waves associated with the DIA and QDIA waves particularly solitary waves (SWs) [26,27] and shock waves [28] have received a great deal of interest in understanding the basic properties of localized electrostatic perturbation in space $[29,30]$ and laboratory dusty plasmas [31-34]. A number of investigations have been made on QDIA SWs [35] and shocks [28] by using K-dV equation. For plasmas with more than two species, it can arise cases where the $\mathrm{K}-\mathrm{dV}$ equation is not valid near a critical value of a certain parameter (say $\mu$ ). The nonlinear term vanishes at this critical value (at $\mu=\mu_{c}$ ) [36] which makes soliton amplitude large enough to break down the validity of K-dV equation. The mmK-dV (mixed modified K-dV) equation, also known as Gardner equation, can give soliton solution around this critical value [37]. The technique of analyzing SWs is Gardner approach which leads to a standard Gardner equation. From the analysis of standard Gardner equation, SW of permanent profile is found, which is known as Gardner soliton (GS) $[1,38,39]$. In our present manuscript, we attempt to study the basic features of QDIA GSs by deriving modified Gardner equation, which is valid around $Z_{d} n_{d 0} / n_{i 0}=2 / 3$, in a quantum dusty plasma containing inertial ions, Fermi electrons with quantum effect, and negatively charged immobile dust. The manuscript is organized as follows. The model equations are provided in Section 2. The Gardner equation is derived by using the reductive perturbation method in Section 3. The analytical solutions are presented in Section 4. A brief discussion is finally given in Section 5 .

\section{Model Equations}

We consider a one-dimensional, collisionless, unmagnetized quantum dusty plasma system composed of inertial ions, massless Fermi electrons with quantum effect, and negatively charged immobile dust. Thus, at equilibrium we have $n_{i 0}=n_{e 0}+Z_{d} n_{d 0}$. The nonlinear dynamics of these low-frequency (purely electrostatic) QDIA waves in such a plasma system is described by the normalized equations of the form

$$
\begin{gathered}
\frac{\partial n_{i}}{\partial t}+\frac{\partial}{\partial x}\left(n_{i} u_{i}\right)=0 \\
\frac{\partial u_{i}}{\partial t}+u_{i} \frac{\partial u_{i}}{\partial x}=-\frac{\partial \phi}{\partial x} \\
\frac{\partial \phi}{\partial x}-\frac{2}{5 n_{e}} \frac{\partial n_{e}^{5 / 3}}{\partial x}+\beta \frac{\partial}{\partial x}\left(\frac{\frac{\partial^{2}}{\partial x^{2}} \sqrt{n_{e}}}{\sqrt{n_{e}}}\right)=0 \\
\frac{\partial^{2} \phi}{\partial x^{2}}=-\rho
\end{gathered}
$$

$$
\rho=n_{i}-(1-\mu) n_{e}-\mu,
$$

where $n_{i}\left(n_{e}\right)$ is the ion (electron) number density normalized by its equilibrium value $n_{i 0}\left(n_{e 0}\right), u_{i}$ is the ion fluid speed normalized by quantum ion-acoustic speed $C_{i}=\left(K_{B} T_{F e} / m_{i}\right)^{1 / 2}$ with $m_{i}$ being the ion rest mass, $K_{B} T_{\mathrm{Fe}}$ is the electron Fermi energy, $K_{B}$ is the Boltzmann constant, and $T_{\mathrm{Fe}}$ is the Fermi temperature of electron, $\phi$ is the electrostatic wave potential normalized by $K_{B} T_{F e} \phi / e$ with $e$ being the magnitude of the charge of an electron, $\rho$ is the normalized surface charge density, and $\mu=Z_{d} n_{d 0} / n_{i 0}$. The time variable $(t)$ is normalized by $\omega_{p i}^{-1}=\left(m_{i} / 4 \pi n_{i 0} e^{2}\right)^{1 / 2}$ and the space variable is normalized by $\lambda_{D i}=\left(K_{B} T_{F e} / 4 \pi n_{i 0} e^{2}\right)^{1 / 2}$. In Equation (3) we have used the following Fermi pressure law for the electron species $[40,41]$

$$
P_{e}=\frac{1}{5} \frac{m_{e} V_{F e}^{2} n_{e}^{5 / 3}}{n_{e 0}^{2 / 3}} .
$$

Also $\beta=\delta H^{2} / 2$ with $\delta=n_{i 0} / n_{e 0}$ and $H=\hbar \omega_{p e} / K_{B} T_{F e}$ is the ratio between the plasmon energy and the electron Fermi energy where

$V_{F e}=\left(2 K_{B} T_{F e} / m_{e}\right)^{1 / 2}$ is the electron Fermi speed at temperature $T_{F e}$.

\section{Gardner Equation}

We first obtain the well known K-dV equation and see why Gardner equation is needed to find SW solution.

\subsection{Derivation of the K-dV Equation}

To obtain the QDIA K-dV equation, we introduce the stretched coordinates

$$
\begin{gathered}
\zeta=\varepsilon^{\frac{1}{2}}\left(x-V_{p} t\right), \\
\tau=\varepsilon^{\frac{3}{2}} t,
\end{gathered}
$$

where $V_{p}$ is the QDIA wave phase speed $(\omega / k)$ and $\varepsilon$ is a smallness parameter measuring the weakness of the dispersion $(0<\varepsilon<1)$. We then expand $n_{i}, n_{e}, u_{i}$, $\phi$, and $\rho$ in power series of $\varepsilon$

$$
\begin{aligned}
& n_{i}=1+\varepsilon n_{i}^{(1)}+\varepsilon^{2} n_{i}^{(2)}+\varepsilon^{3} n_{i}^{(3)}+\cdots \\
& n_{e}=1+\varepsilon n_{e}^{(1)}+\varepsilon^{2} n_{e}^{(2)}+\varepsilon^{3} n_{e}^{(3)}+\cdots \\
& u_{i}=0+\varepsilon u_{i}^{(1)}+\varepsilon^{2} u_{i}^{(2)}+\varepsilon^{3} u_{i}^{(3)}+\cdots \\
& \phi=0+\varepsilon \phi^{(1)}+\varepsilon^{2} \phi^{(2)}+\varepsilon^{3} \phi^{(3)}+\cdots \\
& \rho=0+\varepsilon \rho^{(1)}+\varepsilon^{2} \rho^{(2)}+\varepsilon^{3} \rho^{(3)}+\cdots
\end{aligned}
$$

and develop equations in various powers of $\varepsilon$. To the 
lowest order in $\varepsilon$, Equations (1)-(13) give

$$
\begin{gathered}
u_{i}^{(1)}=\frac{1}{V_{p}} \psi, \\
n_{i}^{(1)}=\frac{1}{V_{p}^{2}} \psi, \\
n_{e}^{(1)}=\frac{3}{2} \psi, \\
V_{p}=\sqrt{\frac{2}{3(1-\mu)},}
\end{gathered}
$$

where $\psi=\phi^{(1)}$. Equation (17) represents the linear dispersion relation for the QDIA waves. This clearly indicates that the QDIA wave phase speed $\left(V_{p}\right)$ increases with the increase of the dust charge density $\left(Z_{d} n_{d 0}\right)$.

To the next higher order of $\varepsilon$, one can obtain another set of coupled equations for $n_{i}^{(2)}, n_{e}^{(2)}$, and $\phi^{(2)}$, which -along with the first set of coupled linear equations for $n_{i}^{(1)}, n_{e}^{(1)}$, and $\phi^{(1)}$-reduce to a nonlinear dynamical equation of the form

$$
\frac{\partial \psi}{\partial \tau}+A B \psi \frac{\partial \psi}{\partial \zeta}+\alpha_{2} \frac{\partial^{3} \psi}{\partial \zeta^{3}}=0,
$$

where

$$
\begin{gathered}
A=\frac{9}{4}(1-\mu)(2-3 \mu), \\
B=\frac{1}{2} V_{p}^{3}, \\
\alpha_{2}=\frac{1}{2} V_{p}^{3}\left(1-\frac{9 H^{2}}{16}\right) .
\end{gathered}
$$

Equation (18) is known as $\mathrm{K}-\mathrm{dV}$ equation. The stationary localized solution of Equation (18) is given by

$$
\psi=\psi_{0} \operatorname{sech}^{2}\left[\left(\zeta-U_{0} \tau\right) / \Delta\right],
$$

where the amplitude $\psi_{0}$ and the width $\Delta$ are given by $\psi_{0}=3 U_{0} / A B$ and $\Delta=\sqrt{4 \alpha_{2} / U_{0}}$, respectively. $U_{0}$ is the mach number. As $U_{0}>0$ and $B>0(\mu<1)$, (22) clearly indicates that 1$)$ small amplitude solitary waves with $\psi>0$, i.e. positive soliton exists if $\mu<2 / 3,2$ ) small amplitude solitary waves with $\psi<0$, i.e. negative soliton exists if $\mu>2 / 3$, and 3) $A=0$ for $\mu=\mu_{c}$ i.e. the nonlinear term vanishes at $\mu=\mu_{c}$ and is not valid near $\mu=\mu_{c}$ which makes soliton amplitude large enough to break down its validity. To find soliton solution around $\mu=2 / 3$, we now obtain gardner equation.

\subsection{Derivation of the Gardner Equation}

To study QDIA GSs by analyzing the ingoing solutions of Equations (1)-(5), we first introduce the stretched co- ordinates [37]

$$
\begin{gathered}
\zeta=\varepsilon\left(x-V_{p} t\right), \\
\tau=\varepsilon^{3} t,
\end{gathered}
$$

By using Equations (23) and (24) in Equations (1)-(6), and Equations (9)-(13) and to the lowest order in $\varepsilon$, we find the same values of $u_{i}^{(1)}, n_{i}^{(1)}, n_{e}^{(1)}$, and $V_{p}$ as like as that of the $\mathrm{K}-\mathrm{dV}$. To the next higher order in $\varepsilon$, we obtain a set of equations, which, after using Equations (14)-(17), can be simplified as

$$
\begin{gathered}
u_{i}^{(2)}=\frac{1}{2 V_{p}^{3}} \psi^{2}+\frac{1}{V_{p}} \phi^{(2)}, \\
n_{i}^{(2)}=\frac{3}{2 V_{p}^{4}} \psi^{2}+\frac{1}{V_{p}^{2}} \phi^{(2)}, \\
n_{e}^{(2)}=\frac{3}{2} \psi^{2}+\frac{9}{8} \phi^{(2)}, \\
\rho^{(2)}=\frac{1}{2} A \psi^{2}=0 .
\end{gathered}
$$

It is obvious from Equation (28) that $A=0$ since $\psi \neq 0$. One can find that $A=0$ at its critical value $\mu=\mu_{c}=2 / 3$ (which is a solution of $A=0$ ). So, for $\mu$ around its critical value $\left(\mu_{c}\right), A=A_{0}$ can be expressed as

$$
A_{0} \simeq S\left(\frac{\partial A}{\partial \mu}\right)_{\mu=\mu_{c}}\left|\mu-\mu_{c}\right|=c_{1} s \varepsilon .
$$

where $c_{1}=-9 / 4,\left|\mu-\mu_{c}\right|$ is a small and dimensionless parameter, and can be taken as the expansion parameter $\varepsilon$, i.e. $\left|\mu-\mu_{c}\right| \simeq \varepsilon$, and $s=1$ for $\mu>\mu_{c}$ and $s=-1$ for $\mu<\mu_{c}$. So, $\rho^{(2)}$ can be expressed as

$$
\varepsilon^{2} \rho^{(2)} \simeq \varepsilon^{3} \frac{1}{2} c_{1} s \psi^{2},
$$

which, therefore, must be included in the third order Poissons equation. To the next higher order in $\varepsilon$, and after some mathematical calculations we obtain a set of equations

$$
\begin{gathered}
\frac{\partial n_{i}^{(3)}}{\partial \zeta}=\frac{15}{2 V_{p}^{6}} \psi^{2} \frac{\partial \psi}{\partial \zeta}+\frac{3}{V_{p}^{4}} \frac{\partial}{\partial \zeta}\left[\psi \phi^{(2)}\right] \\
+\frac{v}{V_{p}^{2} \tau} \psi+\frac{1}{V_{p}^{2}} \frac{\partial \phi^{(3)}}{\partial \zeta}+\frac{2}{V_{p}^{2}} \frac{\partial \psi}{\partial \tau}, \\
\frac{\partial n_{e}^{(3)}}{\partial \zeta}=\frac{81}{16} \psi^{2} \frac{\partial \psi}{\partial \zeta}+\frac{9}{4} \frac{\partial}{\partial \zeta}\left[\psi \phi^{(2)}\right]+\frac{3}{2} \frac{\partial \phi^{(3)}}{\partial \zeta}+\frac{9 \beta}{8} \frac{\partial^{3} \psi}{\partial \zeta^{3}},
\end{gathered}
$$

$$
\frac{\partial^{2} \psi}{\partial \zeta^{2}}=-\frac{1}{2} c_{1} s \psi^{2}-n_{i}^{(3)}+(1-\mu) n_{e}^{(3)} .
$$


Now, combining Equations (31)-(33), we obtain a equation of the form

$$
\frac{\partial \psi}{\partial \tau}+c_{2} s \psi \frac{\partial \psi}{\partial \zeta}+\alpha_{1} \psi^{2} \frac{\partial \psi}{\partial \zeta}+\alpha_{2} \frac{\partial^{3} \psi}{\partial \zeta^{3}}=0
$$

where

$$
\begin{gathered}
c_{2}=\frac{1}{2} c_{1} V_{p}^{3}, \\
\alpha_{1}=\frac{1}{2} V_{p}^{3}\left\{\frac{15}{2 V_{p}^{6}}-\frac{81(1-\mu)}{16}\right\},
\end{gathered}
$$

And $\alpha_{2}$ is given in Equation (21). Equation (34) is known as Gardner equation. It is important to note that if we neglect $\psi^{3}$ term and put $c_{2} s=A B$, the Gardner equation reduces to $\mathrm{K}-\mathrm{dV}$ equation which has derived in Equation (18). However, in this $\mathrm{K}-\mathrm{dV}$ equation the nonlinear term vanishes at $\mu=\mu_{c}$, and is not valid near $\mu=\mu_{c}$ which makes soliton amplitude large enough to break down its validity. But the Gardner equation derived here is valid for $\mu$ near its critical value.

\section{SW Solution of the Gardner Equation}

To analyze stationary GSs, we first introduce a transformation $\xi=\zeta-U_{0} \tau$ which allows us to write Equation (34), under the steady state condition, as

$$
\frac{1}{2}\left(\frac{\mathrm{d} \psi}{\mathrm{d} \xi}\right)^{2}+V(\psi)=0
$$

where the pseudo-potential $V(\psi)$ is

$$
V(\psi)=-\frac{U_{0}}{2 \alpha_{2}} \psi^{2}+\frac{c_{2} s}{6 \alpha_{2}} \psi^{3}+\frac{\alpha_{1}}{12 \alpha_{2}} \psi^{4} .
$$

It is obvious from Equation (38) that

$$
\begin{gathered}
\left.V(\psi)\right|_{\psi=0}=\left.\frac{\mathrm{d} V(\psi)}{\mathrm{d} \psi}\right|_{\psi=0}=0, \\
\left.\frac{\mathrm{d}^{2} V(\psi)}{\mathrm{d} \psi^{2}}\right|_{\psi=0}<0 .
\end{gathered}
$$

The conditions of Equations (39) and (40) imply that SW solutions of (37) exist if

$$
\left.V(\psi)\right|_{\psi=\psi_{m}}=0 .
$$

The latter can be solved as

$$
\begin{aligned}
U_{0} & =\frac{c_{2} s}{3} \psi_{m 1,2}+\frac{\alpha_{1}}{6} \psi_{m 1,2}^{2}, \\
\psi_{m 1,2} & =\psi_{m}\left[1 \mp \sqrt{1+\frac{U_{0}}{V_{0}}}\right],
\end{aligned}
$$

where $\psi_{m}=-c_{2} s / \alpha_{1}$, and $V_{0}=c_{2}^{2} s^{2} / 6 \alpha_{1}$. Now, using
Equations (38) and (43) in Equation (37) we have

$$
\left(\frac{\mathrm{d} \psi}{\mathrm{d} \xi}\right)^{2}+\gamma \psi^{2}\left(\psi-\psi_{m 1}\right)\left(\psi-\psi_{m 2}\right)=0
$$

where $\gamma=\alpha_{1} / 6 \alpha_{2}$. The SW solution of Equations (37) or (44) is, therefore, directly given by

$$
\psi=\left[\frac{1}{\psi_{m 2}}-\left(\frac{1}{\psi_{m 2}}-\frac{1}{\psi_{m 1}}\right) \cosh ^{2}\left(\frac{\xi}{\delta}\right)\right]^{-1},
$$

where $\psi_{m 1,2}$ are given in Equation (43) and SWs width $\delta$ is

$$
\delta=\frac{2}{\sqrt{-\gamma \psi_{m 1} \psi_{m 2}}} .
$$

Figures 1-4 show the variation of amplitude of positive (negative) GSs with $\mu$ for $U_{0}=0.5$ and $H=0.3$. These figures clearly indicate that both positive and negative GSs exist around the crical value, $\mu=2 / 3$. It has been found that the amplitude (magnitude of the amplitude) of both positive and negative GSs decrease with the increase of $\mu$. Figures 2-5 represent the variation of amplitude of positive (negative) GSs with $U_{0}$ for $\mu=0.66$ $(\mu=0.67)$ and $H=0.3$. These figures indicate that the amplitude of both positive and negative GSs increase with the increase of $U_{0}$. We have found that the amplitude of positive and negative GSs does not vary with the quantum diffraction parameter, $H$ but the width of the both positive and negative GSs vary with it. Figures 3-6 imply that the width of both positive and negative GSs decrease with the increase of $H$ and increase with the increase of $\mu$. We have also noticed that in our present system the GSs exist when the quantum effect of electron is neglected.

\section{Discussion}

We have investigated QDIA GSs in quantum dusty plasma by deriving Gardner equation. The K-dV solitons are not valid for $\mu=2 / 3$ and $\mu \sim 2 / 3$, which vanish

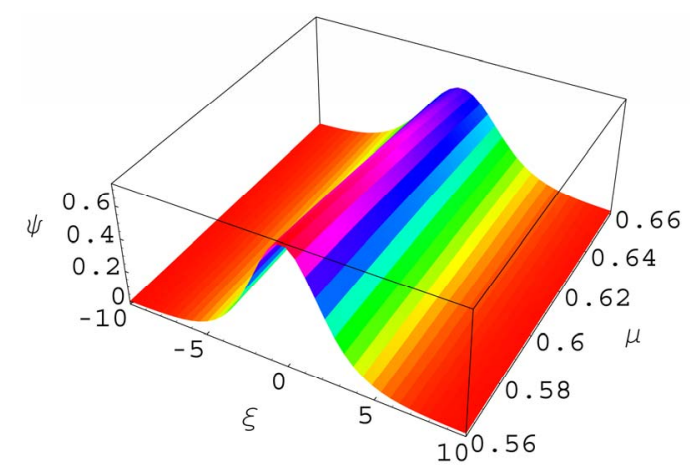

Figure 1. (Color online) Showing the variation of amplitude of the QDIA positive GSs with $\boldsymbol{\mu}$ for $U_{0}=0.5$ and $\boldsymbol{H}=0.3$. 


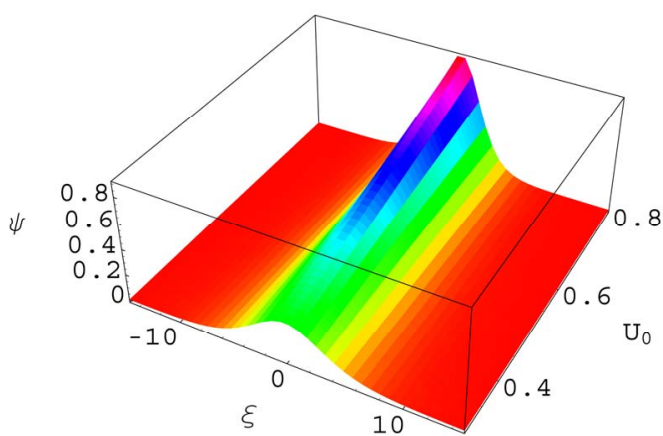

Figure 2. (Color online) Showing the variation of amplitude of the QDIA positive GSs with $U_{0}$ for $\mu=0.66$ and $H=0.3$.

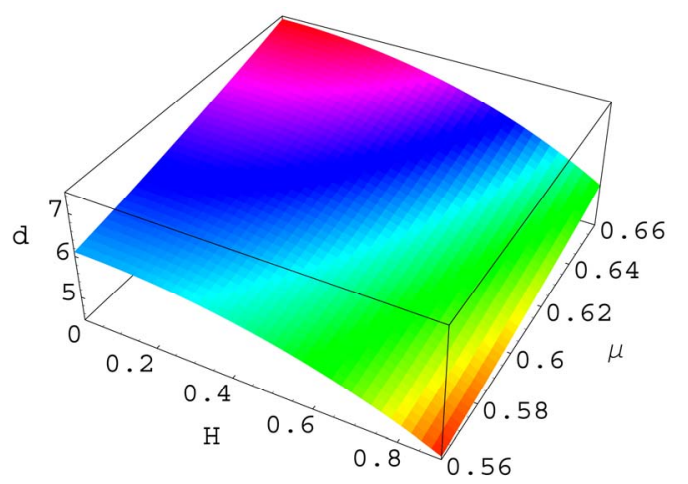

Figure 3. (Color online) Showing the variation of width of the QDIA positive GSs with $H$ and $\mu$ for $U_{0}=0.1$.

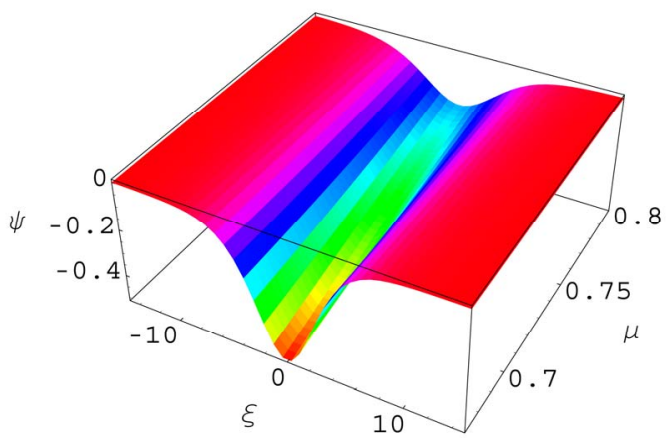

Figure 4. (Color online) Showing the variation of amplitude of the QDIA negative GSs with $\boldsymbol{\mu}$ for $U_{0}=0.5$ and $\boldsymbol{H}=0.3$.

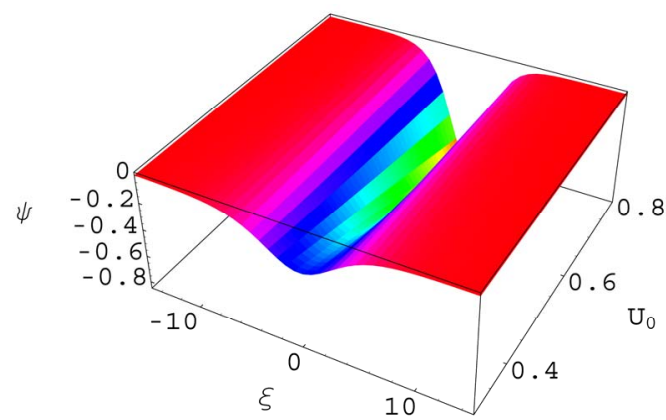

Figure 5. (Color online) Showing the variation of amplitude of the QDIA negative GSs with $U_{0}$ for $\boldsymbol{\mu}=0.67$ and $\boldsymbol{H}=\mathbf{0 . 3}$.

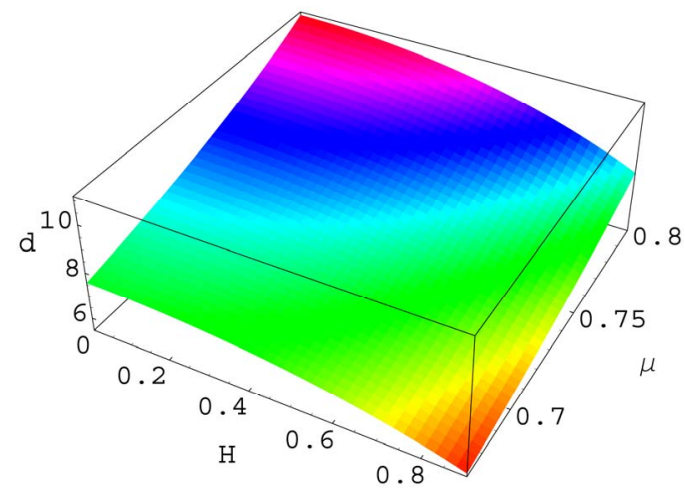

Figure 6. (Color online) Showing the variation of width of the QDIA negative GSs with $H$ and $\mu$ for $U_{0}=0.1$.

at the nonlinear coefficients of the K-dV equation. However, the QDIA GSs investigated in our present work are valid for $\mu \sim 2 / 3$. The results, which have been obtained from this investigation, can be summarized as follows:

1) The quantum dusty plasma system under consideration supports finite amplitude GSs, whose basic features (polarity, amplitude, width, etc.) depend on the ion and dust number densities and quantum diffraction (tunneling) parameter, $H$.

2) GSs are shown to exist for $\mu \sim 2 / 3$, and are found to be different from $\mathrm{K}-\mathrm{dV}$ solitons, which do not exist for $\mu \sim 2 / 3$.

3) It has been found that at $\mu<2 / 3$, positive GSs exist, whereas at $\mu>2 / 3$, negative GSs exist.

4) We have seen that the amplitude of positive and negative GSs decreases with $\mu$, increases with $U_{0}$, and does not depend on $H$.

5) We have also observed that the width of the GSs increases with $\mu$ but decreases with the increase of $H$.

It should be mentioned here that in our present investigation, we have neglected the quantum effect of ions since ions are heavier than electrons. However, QDIA solitary waves in quantum dusty plasma with or without the effects of obliqueness and external magnetic field are also problems of recent interest for many space and laboratory dusty plasma situations, but beyond the scope of our present investigation. In conclusion, we propose that a new experiment may be designed based on our results to observe such waves in both laboratory and space quantum dusty plasma system.

\section{Acknowledgements}

The Third World Academy of Science (TWAS) Research Grant for research equipment is gratefully acknowledged.

\section{REFERENCES}

[1] M. M. Hossain, A. A. Mamun and K. S. Ashrafi, Physics of Plasmas, Vol. 18, 2011, Article ID: 103704. 
http://dx.doi.org/10.1063/1.3646738

[2] A. A. Mamun and P. K. Shukla, Physics Letters A, Vol. 290, 2001, pp. 173-175.

http://dx.doi.org/10.1016/S0375-9601(01)00669-7

[3] Y. D. Jung, Physics of Plasmas, Vol. 8, 2001, p. 3842. http://dx.doi.org/10.1063/1.1386430

[4] G. Chabier, F. Dquchin and Y. Potekhin, Journal of Physics: Condensed Matter, Vol. 14, 2002, p. 133.

[5] M. Opher, L. O. Silvia, D. E. Danger, V. K. Decyk and J. M. Dawson, Physics of Plasmas, Vol. 8, 2001, p. 2454. http://dx.doi.org/10.1063/1.1362533

[6] G. Manfredi, Fields Institute Communications, Plasmas, Vol. 46, 2005, pp. 263-287.

[7] A. Markowich, C. Ringhofer and C. Schmeiser, "Semiconductor Equations," Springer, Vienna, 1990. http://dx.doi.org/10.1007/978-3-7091-6961-2

[8] D. Kremp, Th. Bornath, M. Bonitz and M. Shlanges, Physical Review E, Vol. 60, 1999, pp. 4725-4732. http://dx.doi.org/10.1103/PhysRevE.60.4725

[9] G. Manfredi and M. Feix, Physical Review E, Vol. 53, 1996, pp. 6460-6470. http://dx.doi.org/10.1103/PhysRevE.53.6460

[10] G. Manfredi and F. Haas, Physical Review B, Vol. 64, 2001, Article ID: 075316. http://dx.doi.org/10.1103/PhysRevB.64.075316

[11] D. Anderson, B. Hall, M. Lisak and M. Marklund, Physical Review E, Vol. 65, 2002, Article ID: 046417. http://dx.doi.org/10.1103/PhysRevE.65.046417

[12] P. K. Shukla and S. Ali, Physics of Plasmas, Vol. 13, 2006, Article ID: 112111. http://dx.doi.org/10.1063/1.2390688

[13] M. Marklund, Physics of Plasmas, Vol. 12, 2005, Article ID: 082110. http://dx.doi.org/10.1063/1.2012147

[14] L. G. Garcia, F. Haas, L. P. L. de Oliveira and J. Goedert, Physics of Plasmas, Vol. 12, 2005, Article ID: 012302. http://dx.doi.org/10.1063/1.1819935

[15] S. Mola, G. Manfredi and M. R. Feix, Journal of Physics of Plasmas, Vol. 50, 1993, pp. 145-162. http://dx.doi.org/10.1017/S0022377800026969

[16] F. Haas, L. G. Garcia, J. Goedert and G. Manfredi, Physics of Plasmas, Vol. 10, 2003, p. 3858. http://dx.doi.org/10.1063/1.1609446

[17] N. Suh, M. R. Feix and P. Bertrand, Journal of Computational Physics, Vol. 94, 1991, pp. 403-418. http://dx.doi.org/10.1016/0021-9991(91)90227-C

[18] F. Haas, Physics of Plasmas, Vol. 12, 1993, Article ID: 062117. http://dx.doi.org/10.1063/1.1939947

[19] P. K. Shukla and S. Ali, Physics of Plasmas, Vol. 12, 2005, Article ID: 114502. http://dx.doi.org/10.1063/1.2136376

[20] P. K. Shukla, Physics Letters A, Vol. 352, 2006, pp. 242243. http://dx.doi.org/10.1016/j.physleta.2005.11.065

[21] L. Stenflo, P. K. Shukla and M. Marklund, Europhysics Letters, Vol. 74, 2006, p. 844. http://dx.doi.org/10.1209/epl/i2006-10032-x

[22] P. K. Shukla and V. P. Silin, Physica Scripta, Vol. 45,
2002 , p. 508.

http://dx.doi.org/10.1088/0031-8949/45/5/015

[23] A. Barkan, N. D’Angelo and R. L. Merlino, Planetary and Space Science, Vol. 44, 1996, pp. 239-242. http://dx.doi.org/10.1016/0032-0633(95)00109-3

[24] R. L. Merlino, A. Barkan, C. Thompson and N. D'Angelo, Physics of Plasmas, Vol. 5, 1998, p. 1607. http://dx.doi.org/10.1063/1.872828

[25] K. E. Lonngren, Physics of Plasmas, Vol. 25, 1983, p. 943. http://dx.doi.org/10.1088/0032-1028/25/9/001

[26] W. Masood, A. Mushtaq and R. Khan, Physics of Plasmas, Vol. 14, 2007, Article ID: 123702. http://dx.doi.org/10.1063/1.2803775

[27] S. A. Khan and A. Mushtaq, Physics of Plasmas, Vol. 14, 2007, Article ID: 083703. http://dx.doi.org/10.1063/1.2756752

[28] W. Masood, M. Siddiq, S. Nargis and A. M. Mirza, Physics of Plasmas, Vol. 16, 2009, Article ID: 013705. http://dx.doi.org/10.1063/1.3068171

[29] C. K. Goertz, Reviews of Geophysics, Vol. 27, 1989, pp. 271-292. http://dx.doi.org/10.1029/RG027i002p00271

[30] G. E. Morfill and A. V. Ivlev, Reviews of Modern Physics, Vol. 81, 2009, pp. 1353-1404. http://dx.doi.org/10.1103/RevModPhys.81.1353

[31] R. L. Merlino and J. Goree, Physics Today, Vol. 57, 2004, p. 32. http://dx.doi.org/10.1063/1.1784300

[32] P. K. Shukla and A. A. Mamun, Introduction to Dusty Plasma Physics, IoP Publishing Ltd., Bristol, 2002, 8-27. http://dx.doi.org/10.1887/075030653X

[33] M. Asaduzzaman, "Electro-Acoustic Waves in Presence of Polarization Force," LAP LAMBERT Academic Publishing, Saarbrücken, 2012.

[34] M. Asaduzzaman and A. A. Mamun, Physical Review E, Vol. 86, 2012, Article ID: 016409. http://dx.doi.org/10.1103/PhysRevE.86.016409

[35] S. A. Khan, S. Mahmood and A. M. Mirza, Physics Letters A, Vol. 372, 2009, pp. 148-153. http://dx.doi.org/10.1016/j.physleta.2007.10.062

[36] M. Asaduzzaman and A. A. Mamun, Astrophysics and Space Science, Vol. 341, 2012, pp. 535-542. http://dx.doi.org/10.1007/s10509-012-1102-x

[37] N. C. Lee, Physics of Plasmas, Vol. 16, 2009, Article ID: 042316. http://dx.doi.org/10.1063/1.3121242

[38] M. M. Hossain and A. A. Mamun, Journal of Physics A: Mathematical and Theoretical, Vol. 45, 2012, Article ID: 125501. http://dx.doi.org/10.1088/1751-8113/45/12/125501

[39] M. Hasan, M. M. Hossain and A. A. Mamun, Astrophysics and Space Science, Vol. 345, 2013, pp. 113-118. http://dx.doi.org/10.1007/s10509-013-1384-7

[40] A. Mushtaq and S. A. Khan, Physics of Plasmas, Vol. 14, 2007, Article ID: 052307. http://dx.doi.org/10.1063/1.2727474

[41] L. D. Landau and E. M. Lifshitz, "Statistical Physics, Pt. 1,” Butterworth-Heinemann, Oxford, 1980. 